\title{
ARTICLE
}

Chronic Myeloproliferative Neoplasms

\section{High mortality rate in COVID-19 patients with myeloproliferative neoplasms after abrupt withdrawal of ruxolitinib}

\author{
Tiziano Barbui $\mathbb{D}^{1}$ - Alessandro Maria Vannucchi ${ }^{2}$ - Alberto Alvarez-Larran ${ }^{3}$ - Alessandra lurlo $\mathbb{1}^{4}$. \\ Arianna Masciulli ${ }^{1}$ - Alessandra Carobbio $\mathbb{1}^{1}$ - Arianna Ghirardi ${ }^{1}$ - Alberto Ferrari ${ }^{1}$ - Giuseppe Rossi ${ }^{5}$ - Elena Elli ${ }^{6}$. \\ Marcio Miguel Andrade-Campos ${ }^{7}$. Mercedes Gasior Kabat ${ }^{8} \cdot$ Jean-Jaques Kiladjian $^{9}$. Francesca Palandri ${ }^{10}{ }^{10}$. \\ Giulia Benevolo ${ }^{11}$. Valentin Garcia-Gutierrez ${ }^{12}$ - Maria Laura Fox ${ }^{13}$. Maria Angeles Foncillas $\mathbb{D}^{14}$. \\ Carmen Montoya Morcillo ${ }^{15}$. Elisa Rumi ${ }^{16}$. Santiago Osorio ${ }^{17}$. Petros Papadopoulos ${ }^{18}{ }^{18}$. \\ Massimiliano Bonifacio $\mathbb{1}^{19}$. Keina Susana Quiroz Cervantes ${ }^{20}$ - Miguel Sagues Serrano ${ }^{21}$. \\ Gonzalo Carreno-Tarragona $\mathbb{D}^{22}$ - Marta Anna Sobas ${ }^{23} \cdot$ Francesca Lunghi $^{24}$ - Andrea Patriarca ${ }^{25}$. \\ Begona Navas Elorza ${ }^{26}$. Anna Angona ${ }^{27}$ - Elena Magro Mazo ${ }^{28} \cdot$ Steffen Koschmieder $\mathbb{D}^{29} \cdot$ Marco Ruggeri $^{30}$. \\ Beatriz Cuevas ${ }^{31}$ • Juan Carlos Hernandez-Boluda $\oplus^{32} \cdot$ Emma Lopez Abadia ${ }^{33}$. Blanca Xicoy Cirici ${ }^{34}$. \\ Paola Guglielmelli $\mathbb{D}^{2}$ - Marta Garrote ${ }^{3}$ - Daniele Cattaneo ${ }^{4}$ - Rosa Daffini ${ }^{5}$ - Fabrizio Cavalca ${ }^{6}$. Beatriz Bellosillo ${ }^{7}$. \\ Lina Benajiba ${ }^{9} \cdot$ Natalia Curto-Garcia ${ }^{35} \cdot$ Marta Bellini $^{36} \cdot$ Silvia Betti ${ }^{37}$ - Valerio De Stefano $\mathbb{1}^{37} \cdot$ Claire Harrison $^{35}$. \\ Alessandro Rambaldi $\mathbb{D}^{36,38}$
}

Received: 7 October 2020 / Revised: 29 October 2020 / Accepted: 27 November 2020 / Published online: 7 January 2021

(c) The Author(s), under exclusive licence to Springer Nature Limited 2021

\begin{abstract}
We report the clinical presentation and risk factors for survival in 175 patients with myeloproliferative neoplasms (MPN) and COVID-19, diagnosed between February and June 2020. After a median follow-up of 50 days, mortality was higher than in the general population and reached $48 \%$ in myelofibrosis (MF). Univariate analysis, showed a significant relationship between death and age, male gender, decreased lymphocyte counts, need for respiratory support, comorbidities and diagnosis of MF, while no association with essential thrombocythemia (ET), polycythemia vera (PV), and prefibrotic-PMF (pre-PMF) was found. Regarding MPN-directed therapy ongoing at the time of COVID-19 diagnosis, Ruxolitinib (Ruxo) was significantly more frequent in patients who died in comparison with survivors $(p=0.006)$. Conversely, multivariable analysis found no effect of Ruxo alone on mortality, but highlighted an increased risk of death in the 11 out of 45 patients who discontinued treatment. These findings were also confirmed in a propensity score matching analysis. In conclusion, we found a high risk of mortality during COVID-19 infection among MPN patients, especially in MF patients and/or discontinuing Ruxo at COVID-19 diagnosis. These findings call for deeper investigation on the role of Ruxo treatment and its interruption, in affecting mortality in MPN patients with COVID-19.
\end{abstract}

\section{Introduction}

The SARS-CoV-2 coronavirus infection causing the coronavirus 2019 disease (COVID-19) is a highly contagious disease that appeared in China in December 2019 and is

Supplementary information The online version of this article (https:// doi.org/10.1038/s41375-020-01107-y) contains supplementary material, which is available to authorized users.

Tiziano Barbui

tbarbui@fondazionefrom.it

Extended author information available on the last page of the article now extensively spreaded to multiple European and extraEuropean countries. The clinical spectrum of SARS-CoV-2 infection appears to be wide, encompassing asymptomatic infection, mild upper respiratory tract illness, and severe interstitial pneumonia with respiratory failure and high risk of death [1]. COVID-19 infected patients are also likely to be at increased risk of venous and arterial thromboembolism, as reported in China [2] and confirmed in autopsies of patients experiencing sudden death [3].

This infection has spread to Europe in February 2020 and, since then, thousands of studies addressing various aspects of COVID-19 have started and numerous clinical trials have been registered on ClinicalTrials.gov. However, there is 
limited information describing the presenting characteristics and outcomes of COVID-19 patients with myeloproliferative neoplasms (MPN), including essential thrombocythemia (ET), polycythemia vera (PV), and myelofibrosis (MF) $[4,5]$. Natural history of MPN is marked by a high incidence of thrombosis and hemorrhagic complications and by a natural propensity to transform into overt MF and acute leukemia. Compared to the general population, overall expected survival is reduced, particularly in overt MF where it ranges from 2 to 5 years, depending on the stage of the disease [6].

MPN experts appointed by the American Society of Hematology (ASH) are updating their answers to some frequently asked clinical questions (https://www.hema tology.org/COVID-19/COVID-19-and-myeloproliferativeneoplasms) regarding management of these patients under COVID-19; in particular, they discuss the role of antithrombotic drugs and how to handle the MPN-directed cytoreductive therapy before and after the onset of coronavirus infection. However, it should be acknowledged that these recommendations are derived from a consensus among experts in the MPN field, and that information on the real-world clinical practice of patients with MPN and CPVID-19 needs to be collected in order to assess the magnitude of the main outcomes' frequency, such as survival and the corresponding risk factors, in the single MPN phenotype.

With this purpose, in May 2020, the European Leukemia Net (ELN), launched an observational study in Europe involving 38 centers from Italy, Spain, Germany, Poland, France, and United Kingdom. Here we report the first results of this study, focussing on clinical/laboratory presentation and risk factors for overall survival during the acute phase of SARS-CoV-2 infection.

\section{Methods}

\section{Patients and COVID-19 diagnosis}

This observational retrospective study (MPN-COVID) was promoted by ELN and had the endorsement of the European Haematology Association, GEMFIN Spanish network on MPN and HARMONY platform.

MPN-COVID study involved 180 patients recruited from 38 European hematology units. Sponsor of the study is the Foundation for Research (FROM) at Papa Giovanni XXIII Hospital in Bergamo, Italy, that obtained the approval by the Italian National Ethical Committee (Spallanzani Hospital, in Rome); all other centers had the approval by their respective local ethical committees.

We included consecutive adult patients with WHO-2016 diagnosed MPN from Spain $(n=81)$, Italy $(n=77)$, UK $(n=11)$, France $(n=7)$, Poland $(n=3)$, Germany $(n=1)$.
Five patients were excluded from the analysis: three for acute leukaemia post-MPN and two for excess missing data. Therefore, the present analysis comprises 175 patients. Written informed consent of participants was collected where possible according to each Country regulation.

SARS-CoV-2 infection was ascertained by a positive real-time reverse transcriptase polymerase chain reaction from nasal swab $(n=155,88.6 \%)$ : for patients unable to swab ( $n=20,11.4 \%$, COVID-19 diagnosis was based on presence of pneumonia and symptoms highly suggestive for COVID-19, such as fever, cough, oxygen saturation $\left(\mathrm{O}_{2}\right.$ saturation) $\leq 93 \%$ at rest, dyspnea, diarrhea.

\section{Data collection and outcome}

The participating centers were asked to report in an electronic case report form) their consecutive MPN patients who developed COVID-19 in the period between 15 February 2020 and 31 May 2020. Data collected included patient demographics, comorbidities, medications, initial laboratory tests, chest radiographs, or CT scan prescribed by the treating physician, drug treatment for COVID-19, invasive mechanical ventilation, or noninvasive respiratory support in hospitalized general wards and intensive care unit (ICU) or in patients managed at home.

The additional information about MPN disease status concerned driver mutations (JAK2V617F, CALR and MPL), duration of MPN before COVID-19, presence of splenomegaly and cytoreductive drugs.

The study outcome was mortality from all-causes and was registered in hospitalized patients (regular wards or ICU) and in patients managed at home.

\section{Statistical methods}

Statistical analysis was carried out at the bio-statistical laboratory of the Foundation for Research (FROM) at Papa Giovanni XXIII Hospital in Bergamo.

Continuous variables were summarized with median along with interquartile range (IQR), and categorical ones were presented as frequencies and percentages. Characteristics of the study population were stratified for survival and differences between the two groups tested with the $\chi^{2}$ test (or Fisher's exact test where appropriate) or rank-sum test for categorical or continuous variables, respectively.

Overall survival was estimated by Kaplan-Meier method and compared according to Country, MF diagnosis, patient disposition, and Ruxolitinib (Ruxo) exposure and discontinuation with the $\log$ rank test. Using a multivariable logistic regression model, association with overall survival was evaluated for the following variables: age, sex, MF diagnosis, chronic heart disease, need of respiratory support, ICU admission, Ruxo exposure, and discontinuation. 
A propensity score (PS) matching analysis [7] was performed to balance patients treated or not with Ruxo, by forming matched sets of 1 treated and 1 randomly-sampled non-treated patient (1:1 matching) who shared similar PS. The PS was estimated by logistic regression of exposure to Ruxo on the baseline covariates (MPN diagnosis, age, haemoglobin, neutrophilis/lymphocytes, platelets count, duration of MPN at COVID-19 onset, hospitalization for COVID-19). Matching was done using the nearest neighbor method with replacement and with caliper of width equal to 0.2 of the pooled standard deviation of the PS logit.

For all tested hypotheses, two-tailed $p$ values $<0.05$ were considered significant. Statistical analysis was performed using STATA Software, release 16 (StataCorp LP, College Station TX, USA).

\section{Results}

\section{Survival and patient characteristics at baseline by mortality}

After diagnosis of COVID-19, 77\% of the patients were hospitalized and $23 \%$ were followed at home; of the 59.2\% who required respiratory support, $47.7 \%$ were managed with noninvasive respiratory support while 19 (11\%) required ICU management. Overall, after a median followup of 50 days, 50 of the 175 MPN patients (28.6\%) died at a median time of 9.5 days after diagnosis (Fig. 1A). This figure was not different among the participating Countries; in Italy and Spain, which were the major contributors of patients (44\% and $44 \%$, respectively), and in the rest of
Europe (12\% of cases) mortality was $32 \%, 25 \%$, and $29 \%$, respectively (Fig. 1B). Reported causes of death were multiorgan failure $(42 \%)$, pneumonia $(33 \%)$, or undefined (25\%).

Table 1 shows characteristics of MPN patients stratified by survival. The diagnosis of ET, PV and pre-PMF did not influence the proportion of survivors versus non-survivors, while the MF phenotype was associated with a greater percentage of non-surviving patients $(48.0 \%$ vs. $28.8 \%$; $p=0.016$ ). Figure $2 \mathrm{~A}$ illustrates the probability of death in MF vs. the other phenotypes. Survival did not differ as regards to driver mutations, palpable splenomegaly, duration of MPN disease, or blood counts, with the exception of a decreased lymphocyte count and a higher neutrophil/ lymphocyte ratio that was registered in non-survivors. Similar to what reported in general population, chronic heart failure, diabetes, older age, oxygen saturation at presentation, need of hospitalization, non-invasive and invasive respiratory support and need of being transferred to ICU were all associated with significantly lower proportion of survivors. In Fig. 2B, the survival curves in MPN patients according to the patient disposition are shown. As expected, the rate of death in ICU patients was 50\%, compared to $25 \%$ and $6 \%$ for cases followed in the regular ward and at home, respectively $(p<0.001)$.

\section{Role of MPN-directed therapy on survival}

At the time of COVID-19 diagnosis, $45.1 \%$ of the patients were receiving hydroxyurea (HU), $25.7 \%$ ruxolitinib, $2.3 \%$ interferon, $4.6 \%$ anagrelide, and $2.3 \%$ other drugs, while $19.4 \%$ were not receiving MPN-directed therapies.
Fig. 1 Overall survival. Kaplan Meier estimates for the overall survival (A), stratified by Country (B). $P$-values are calculated by log-rank test. CI confidence interval.
A

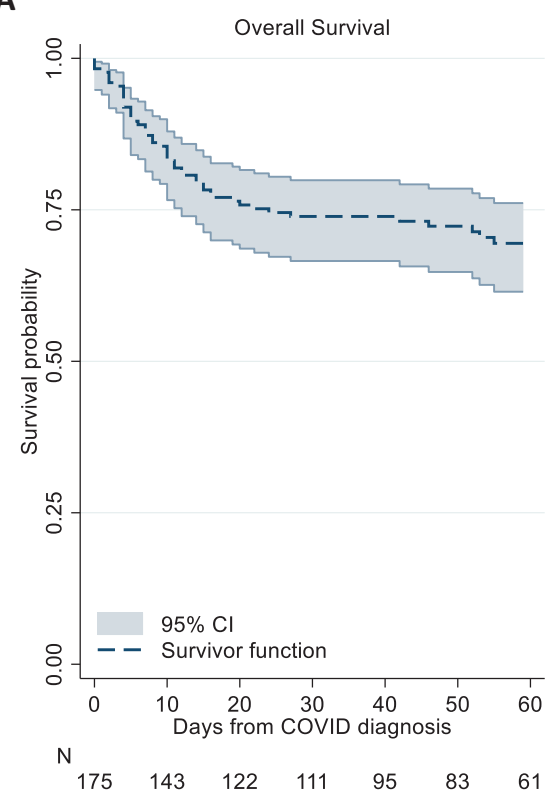

B

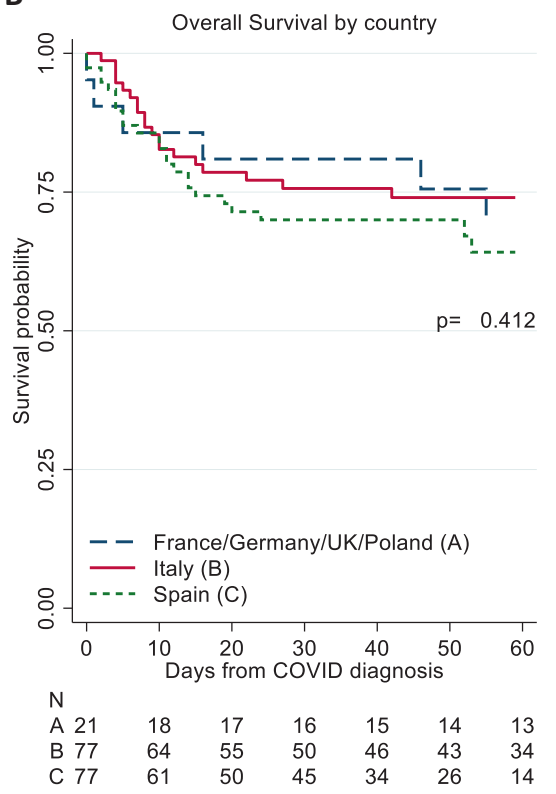


Table 1 MPN-COVID clinical and laboratory characteristics in survivors and non-survivors.

\begin{tabular}{|c|c|c|c|c|c|}
\hline & $\begin{array}{l}N \text { non- } \\
\text { missing }\end{array}$ & $\begin{array}{l}\text { TOTAL } \\
N=175\end{array}$ & $\begin{array}{l}\text { Survivors } \\
N=125\end{array}$ & $\begin{array}{l}\text { Non-survivors } \\
N=50\end{array}$ & $p$ value \\
\hline Sex (female/male), $n(\%)$ & 175 & $73 / 102(41.7 \% / 58.3 \%)$ & $58 / 67(46.4 \% / 53.6 \%)$ & $15 / 35(30.0 \% / 70.0 \%)$ & 0.062 \\
\hline Age, years, median (IQR) & 174 & $71.0(60.0-79.9)$ & $69.5(57.6-78.9)$ & $74.1(69.6-82.1)$ & 0.001 \\
\hline$<60$ years, $n(\%)$ & & $42(24.1 \%)$ & $40(32.0 \%)$ & $2(4.1 \%)$ & $<0.001$ \\
\hline $60-70$ years, $n(\%)$ & & $37(21.3 \%)$ & $24(19.2 \%)$ & $13(26.5 \%)$ & \\
\hline$>70$ years, $n(\%)$ & & $95(54.6 \%)$ & $61(48.8 \%)$ & $34(69.4 \%)$ & \\
\hline MPN type, $n(\%)$ & 175 & & & & \\
\hline Essential thrombocythemia & & $51(29.1 \%)$ & $38(30.4 \%)$ & $13(26.0 \%)$ & 0.56 \\
\hline Polycythemia vera & & $46(26.3 \%)$ & 37 (29.6\%) & $9(18.0 \%)$ & 0.12 \\
\hline Myelofibrosis & & $60(34.3 \%)$ & $36(28.8 \%)$ & $24(48.0 \%)$ & 0.016 \\
\hline $\begin{array}{l}\text { Prefibrotic primary } \\
\text { myelofibrosis }\end{array}$ & & $18(10.3 \%)$ & $14(11.2 \%)$ & $4(8.0 \%)$ & 0.53 \\
\hline $\begin{array}{l}\text { Time from MPN to COVID-19 } \\
\text { diagnosis, years, median (IQR) }\end{array}$ & 174 & $6.0(3.1-11.0)$ & $6.0(3.1-10.4)$ & $5.8(3.2-11.5)$ & 0.81 \\
\hline JAK2V617F, $n(\%)$ & 170 & $123(72.4 \%)$ & $90(73.2 \%)$ & $33(70.2 \%)$ & 0.70 \\
\hline CALR, $n(\%)$ & 95 & $27(28.4 \%)$ & $20(30.3 \%)$ & $7(24.1 \%)$ & 0.54 \\
\hline MPL, $n(\%)$ & 90 & $5(5.6 \%)$ & $2(3.2 \%)$ & $3(10.7 \%)$ & 0.15 \\
\hline Palpable splenomegaly, $n(\%)$ & 153 & $41(26.8 \%)$ & $28(26.2 \%)$ & $13(28.3 \%)$ & 0.92 \\
\hline \multicolumn{6}{|l|}{ MPN drugs, $n(\%)$} \\
\hline Hydroxyurea & 175 & $79(45.1 \%)$ & $60(48.0 \%)$ & $19(38.0 \%)$ & 0.23 \\
\hline $\begin{array}{l}\text { Discontinued after COVID- } \\
19 \text { onset }\end{array}$ & 52 & $9(17.3 \%)$ & $7(21.2 \%)$ & $2(10.5 \%)$ & 0.33 \\
\hline Ruxolitinib & 175 & $45(25.7 \%)$ & $25(20.0 \%)$ & $20(40.0 \%)$ & 0.006 \\
\hline $\begin{array}{l}\text { Discontinued after COVID- } \\
19 \text { onset }\end{array}$ & 45 & $11(24.4 \%)$ & $2(8.0 \%)$ & $9(45.0 \%)$ & 0.004 \\
\hline Interferon & 175 & $4(2.3 \%)$ & $4(3.2 \%)$ & $0(0.0 \%)$ & 0.20 \\
\hline Anagrelide & 175 & $8(4.6 \%)$ & $5(4.0 \%)$ & $3(6.0 \%)$ & 0.57 \\
\hline Other & 175 & $5(2.3 \%)$ & $4(3.2 \%)$ & $1(2.0 \%)$ & 0.67 \\
\hline \multicolumn{6}{|l|}{ Comorbidities, $n(\%)$} \\
\hline Cerebrovascular disease & 174 & $23(13.2 \%)$ & $17(13.7 \%)$ & $6(12.0 \%)$ & 0.76 \\
\hline $\begin{array}{l}\text { Chronic dialysis/Kidney } \\
\text { disease }\end{array}$ & 174 & $19(10.9 \%)$ & $9(7.3 \%)$ & $10(20.0 \%)$ & 0.015 \\
\hline Chronic heart failure & 173 & $25(14.5 \%)$ & $13(10.6 \%)$ & $12(24.0 \%)$ & 0.023 \\
\hline $\begin{array}{l}\text { Chronic obstructive } \\
\text { pulmonary disease }\end{array}$ & 174 & $25(14.4 \%)$ & $16(12.9 \%)$ & $9(18.0 \%)$ & 0.39 \\
\hline $\begin{array}{l}\text { Current/former } \\
\text { tobacco smoker }\end{array}$ & 152 & $35(23.0 \%)$ & $22(20.2 \%)$ & $13(30.2 \%)$ & 0.19 \\
\hline Hyperlipidemia & 168 & $47(28.0 \%)$ & $36(29.8 \%)$ & $11(23.4 \%)$ & 0.41 \\
\hline Hypertension & 171 & $104(60.8 \%)$ & $70(57.9 \%)$ & $34(68.0 \%)$ & 0.22 \\
\hline Diabetes mellitus & 172 & $23(13.4 \%)$ & $12(9.8 \%)$ & $11(22.4 \%)$ & 0.027 \\
\hline \multicolumn{6}{|c|}{ Blood values at COVID-19 diagnosis, median (IQR) } \\
\hline Hemoglobin, g/dL & 145 & $12.4(10.0-13.6)$ & $12.6(10.4-13.5)$ & $11.7(9.5-13.9)$ & 0.37 \\
\hline White blood cells, $\times 10^{9} / \mathrm{L}$ & 145 & $6.5(4.6-10.1)$ & $6.4(4.5-9.2)$ & $7.1(5.0-11.7)$ & 0.14 \\
\hline Lymphocytes, $\times 10^{9} / \mathrm{L}$ & 130 & $0.9(0.6-1.6)$ & $0.9(0.6-1.6)$ & $0.7(0.6-1.1)$ & 0.098 \\
\hline $\begin{array}{l}\text { Neutrophils/ } \\
\text { lymphocytes ratio }\end{array}$ & 125 & $5.4(3.4-8.9)$ & $5.0(3.3-8.6)$ & $7.3(4.5-9.9)$ & 0.029 \\
\hline Platelets, $\times 10^{9} / \mathrm{L}$ & 143 & $252.0(152.0-394.0)$ & $260.5(170.5-437.5)$ & $245.0(121.0-338.0)$ & 0.14 \\
\hline $\mathrm{O}_{2}$ saturation, $\%$, median (IQR) & 114 & $93.0(88.0-96.0)$ & $94.0(91.0-96.0)$ & $90.0(88.0-93.0)$ & $<0.001$ \\
\hline Disposition, $n(\%)$ & 175 & & & & $<0.001$ \\
\hline Home & & $40(22.9 \%)$ & $38(30.4 \%)$ & $2(4.0 \%)$ & \\
\hline Regular ward & & $116(66.3 \%)$ & $80(64.0 \%)$ & $36(72.0 \%)$ & \\
\hline Intensive care unit & & $19(10.9 \%)$ & $7(5.6 \%)$ & $12(24.0 \%)$ & \\
\hline \multicolumn{6}{|l|}{ Main symptoms, $n(\%)$} \\
\hline Fever & 175 & $140(80.0 \%)$ & $99(79.2 \%)$ & $41(82.0 \%)$ & 0.68 \\
\hline Dispnea & 175 & $97(55.4 \%)$ & $66(52.8 \%)$ & $31(62.0 \%)$ & 0.27 \\
\hline \multirow[t]{2}{*}{ Gastrointestinal } & 175 & $22(12.6 \%)$ & $12(9.6 \%)$ & $10(20.0 \%)$ & 0.061 \\
\hline & 174 & $103(59.2 \%)$ & $59(47.2 \%)$ & $44(89.8 \%)$ & $<0.001$ \\
\hline
\end{tabular}


Table 1 (continued)

\begin{tabular}{|c|c|c|c|c|c|}
\hline & $\begin{array}{l}N \text { non- } \\
\text { missing }\end{array}$ & $\begin{array}{l}\text { TOTAL } \\
N=175\end{array}$ & $\begin{array}{l}\text { Survivors } \\
N=125\end{array}$ & $\begin{array}{l}\text { Non-survivors } \\
N=50\end{array}$ & $p$ value \\
\hline \multicolumn{6}{|l|}{$\begin{array}{l}\text { Need of respiratory support, } \\
n(\%)\end{array}$} \\
\hline None & & $71(40.8 \%)$ & $66(52.8 \%)$ & $5(10.2 \%)$ & $<0.001$ \\
\hline Non-invasive & & $83(47.7 \%)$ & $53(42.4 \%)$ & $30(61.2 \%)$ & \\
\hline Invasive & & $20(11.5 \%)$ & $6(4.8 \%)$ & $14(28.6 \%)$ & \\
\hline \multicolumn{6}{|l|}{ COVID-19 drugs, $n(\%)$} \\
\hline Steroid & 162 & $45(27.8 \%)$ & $28(23.7 \%)$ & $17(38.6 \%)$ & 0.060 \\
\hline Antibiotic & 162 & $114(70.4 \%)$ & $77(65.8 \%)$ & $37(82.2 \%)$ & 0.040 \\
\hline Hydroxychloroquine & 166 & $100(60.2 \%)$ & $73(59.8 \%)$ & $27(61.4 \%)$ & 0.86 \\
\hline Antiviral & 166 & $57(34.3 \%)$ & $43(35.8 \%)$ & $14(30.4 \%)$ & 0.51 \\
\hline Lopinavir/Ritonavir & 53 & $46(86.8 \%)$ & $33(82.5 \%)$ & $13(100.0 \%)$ & 0.17 \\
\hline Other & 53 & $7(13.2 \%)$ & $7(17.5 \%)$ & $0(0.0 \%)$ & \\
\hline Experimental & 170 & $19(11.2 \%)$ & $11(8.9 \%)$ & $8(17.0 \%)$ & 0.13 \\
\hline Tocilizumab & 19 & $15(78.9 \%)$ & $7(63.6 \%)$ & $8(100.0 \%)$ & 0.39 \\
\hline Ruxolitinib & 19 & $2(10.5 \%)$ & $2(18.2 \%)$ & $0(0.0 \%)$ & \\
\hline Other & 19 & $2(10.5 \%)$ & $2(18.2 \%)$ & $0(0.0 \%)$ & \\
\hline Antithrombotic & 166 & $93(56.0 \%)$ & $70(57.9 \%)$ & $23(51.1 \%)$ & 0.44 \\
\hline Direct oral anticoagulants & 92 & $2(2.2 \%)$ & $2(2.9 \%)$ & $0(0.0 \%)$ & 1.00 \\
\hline $\begin{array}{l}\text { Low molecular weight } \\
\text { heparin }\end{array}$ & 92 & $89(96.7 \%)$ & $66(95.7 \%)$ & $23(100.0 \%)$ & \\
\hline Warfarin & 92 & $1(1.1 \%)$ & $1(1.4 \%)$ & $0(0.0 \%)$ & \\
\hline
\end{tabular}

Univariate analysis.

$M P N$ myeloproliferative neoplasms.

Stratification of MPN-directed ongoing treatment at COVID-19 diagnosis by survival category revealed a significantly higher proportion of non-survivors $(40 \%)$ among the 45 patients (ET $n=2$; PV $n=8$; MF $n=34$; pre-PMF $n=1)$ who were on Ruxo $(p=0.006)$.

After COVID-19 diagnosis, $11.4 \%$ and $24.4 \%$ of the patients on HU and Ruxo, respectively, stopped treatment. Of note, median time from COVID-19 diagnosis to Ruxo discontinuation was 0.5 days, i.e. immediately subsequent to diagnosis. By analysing survival of patients who continued vs. those who stopped Ruxo therapy during the infection course, we found that the latter had higher mortality (Fig. 3A). Survival probabilities at 60 days from COVID-19 diagnosis were $75 \%, 68 \%$, and $11 \%$ in no Ruxo, Ruxo continued, and Ruxo discontinued groups, respectively $(p<0.001)$. We found no such difference for the patients who stopped HU after COVID-19 diagnosis.

\section{Risk factors for mortality}

We included in a logistic multivariable model the most relevant risk factors for survival found in univariate analysis, i.e. age, sex, MF phenotype, chronic heart disease, need of respiratory support, ICU admission, Ruxo treatment and Ruxo discontinuation during the coronavirus infection. Of these, age $(\mathrm{OR}=1.07, p=0.003)$, male gender $(\mathrm{OR}=$ $2.48, p=0.047)$, admission to ICU (OR $=3.6, p=0.037)$, need of respiratory support $(\mathrm{OR}=10.4, p<0.001)$ and Ruxo discontinuation ( $\mathrm{OR}=8.4, p=0.040)$ were statistically significant; however, some confidence intervals (CIs), in particular the one for Ruxo discontinuation, were wide, likely due to the low number of available events compared to number of confounders (Table 2). Nevertheless, these significances were statistically robust to inclusion of both the known relevant COVID-19-related and patient-related confounders.

Moreover, to provide more robust evidence of this finding, we performed a sensitivity analysis by PS matching. Baseline characteristics before and after PS matching (Table S1) showed that those factors linked to the severity of MPN and those associated with COVID-19 were well balanced in the two groups. Patients for whom no match was found were excluded, leading to a reduced number of matched pairs (22 exposed/nonexposed pairs). The overall picture of comparative profiles is well illustrated in Fig. 3B, confirming the negative effect of Ruxo discontinuation found in the analysis on the full patient set (Fig. 3A).

\section{Discussion}

In this multicenter European study, we describe a relatively large MPN series of 175 patients with MPN and COVID-19, collected under conditions of exceptional COVID-19 lethality, from February to June 2020, and report estimates and 
Fig. 2 Survival by MPN phenotypes and patients disposition. Kaplan Meier survival estimates stratified by MPN phenotypes (A) and patients disposition (B). $P$ values are calculated by log-rank test. MF myelofibrosis, MPNs myeloproliferative neoplasms, ICU intensive care unit.
Fig. 3 Survival by Ruxolitinib exposure and discontinuation. Kaplan Meier survival estimates stratified by the use and discontinuation of Ruxo in the full analysis set (A) and after PS matching (B). $P$-values are calculated by log-rank test. PS propensity score, Ruxo ruxolitinib.

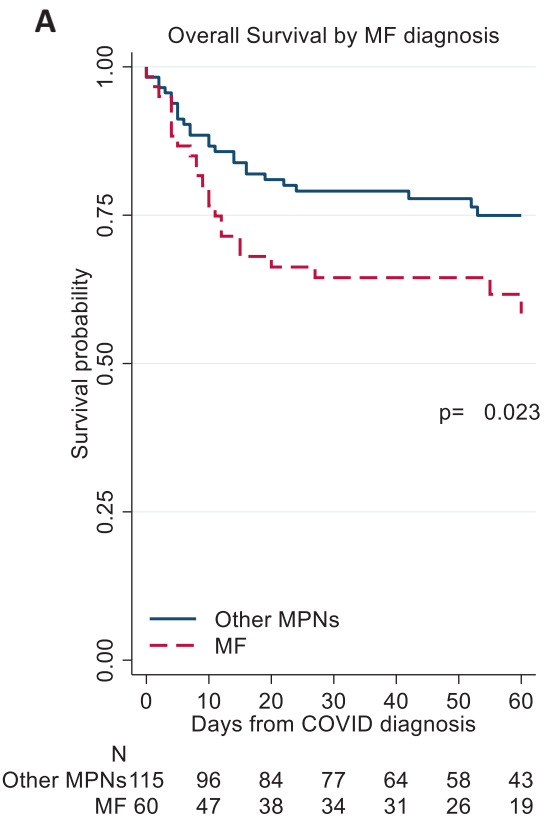

B
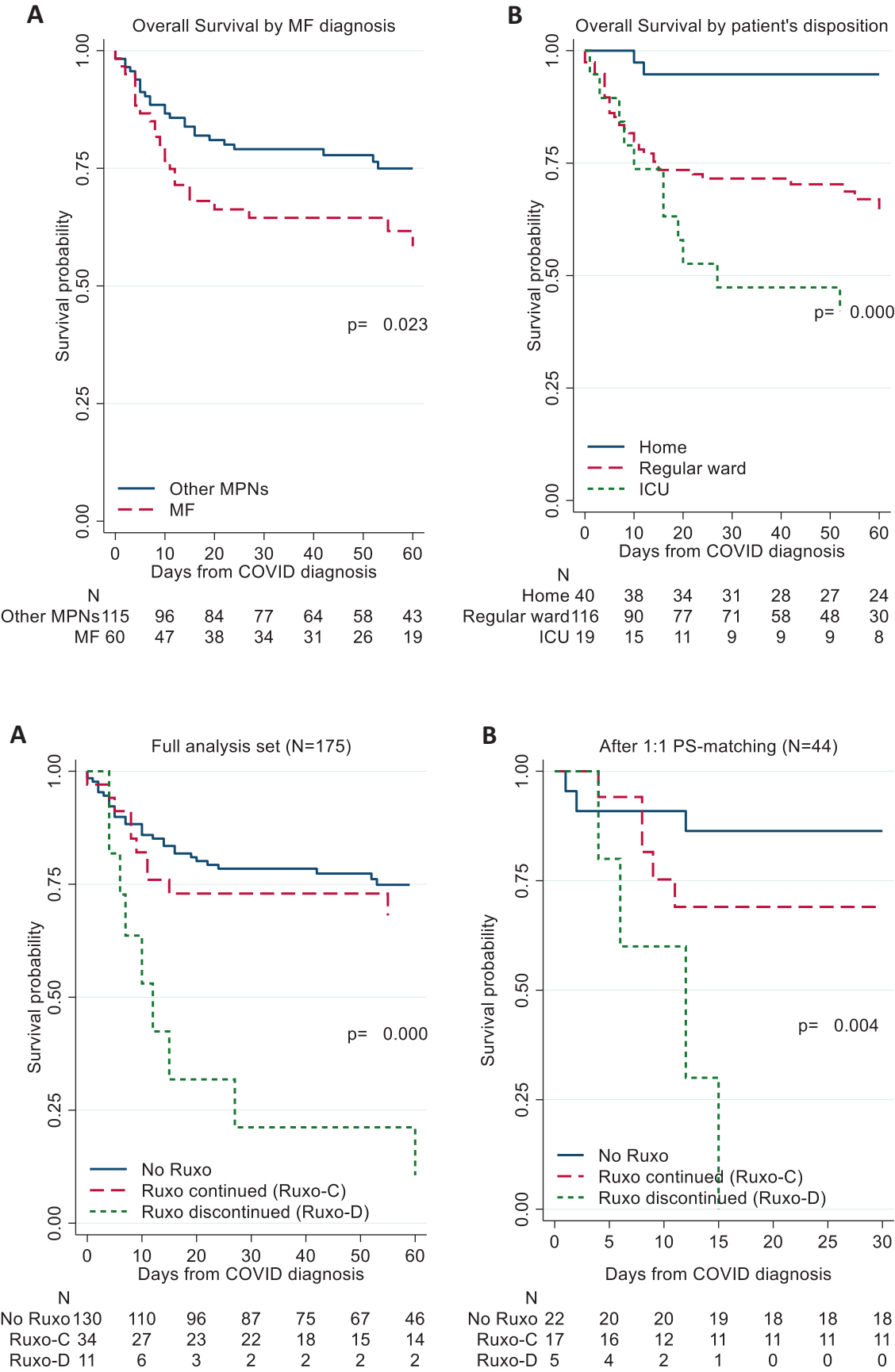

risk factors of mortality. In our sample, death occurred more frequently among males, and, as expected, in older aged patients. Of note, MF was the most represented among the MPNs (34\%) and HU was the most common treatment $(45.1 \%)$. Ruxo was used in $25 \%$ of cases, a figure that is consistent with recent reports on real-world clinical treatment of MPNs [8, 9] and in agreement with the high prevalence of MF in our cohort.

All the 135 hospitalized patients and 20 (50\%) of those treated at home had COVID-19 diagnosis confirmed by a positive result on polymerase chain reaction testing of a nasopharyngeal sample; the remaining 20 patients managed at home were diagnosed based on highly suggestive symptomatology. In this regard, it should be noted that during the period in which the study was conducted, the possibility of carrying out diagnostic tests with nasopharyngeal swab was limited, especially in patients at home.

Concerning COVID-19 presentation and therapy, our MPN patients did not differ from the general non-MPN population under the profile of inflammatory markers or comorbidities, and drug treatment was consistent with therapeutic standards at the time. In particular, the use of steroids 
Table 2 Multivariable analysis of risk factors for mortality after COVID-19 diagnosis.

\begin{tabular}{llr}
\hline & OR $(95 \% \mathrm{CI})$ & $p$ value \\
\hline Age & $1.07(1.02-1.11)$ & 0.003 \\
Male sex & $2.48(1.01-6.07)$ & 0.047 \\
MF diagnosis & $1.69(0.61-4.66)$ & 0.315 \\
Chronic heart disease & $2.18(0.64-7.36)$ & 0.210 \\
Respiratory support & $10.0(2.94-34.0)$ & $<0.001$ \\
ICU admission & $4.93(1.36-17.9)$ & 0.015 \\
Ruxolitinib administration & $2.40(0.72-8.02)$ & 0.154 \\
Ruxolitinib discontinuation & $8.51(1.14-63.4)$ & 0.037 \\
\hline
\end{tabular}

$O R$ odds ratio, $C I$ confidence interval, $M F$ myelofibrosis, ICU intensive care unit.

was very limited. Estimates of mortality were higher than in the general population and did not differ by Country. Comparable figures of mortality were reported in a recent Italian study on COVID-19 and hematological malignancies, in which 27 of 83 patients with MPN died (33\%) [10]. Notably, mortality in our patients with MF was higher (48\%) in comparison to ET, PV, or pre-PMF ( $25 \%)$, suggesting a higher vulnerability of patients with MF to COVID-19. As expected, the highest rate of mortality was in patients admitted to ICU (50\%), while it was only 25 and $6 \%$ in patients admitted to regular ward or at home. When compared with mortality rate found in the general population in Italy and Spain, the two Countries with the highest number of patients in this study, these rates exceed the overall case-fatality rate reported in Italy $(12.8 \%)$ in the same period and are comparable to those observed in Spain (23\%) only for ET, PV, and pre-PMF but not for MF $(48 \%)[11,12]$.

An unexpected result of this study was the association between Ruxo and overall mortality that was found in $40 \%$ of the 45 cases treated with this drug. In further investigations by multivariable analysis and PS matching, we highlighted that this effect was due not so much to drug exposure but to its rapid discontinuation (median 0.5 days), that accounted for $75 \%$ of deaths. In fact, in a multivariable analysis we identified a set of variables that were significantly correlated with mortality, even adjusting for known confounders (age, gender, respiratory support and admission to ICU). Contrary to what observed in univariate analysis, in logistic regression we found no effect of Ruxo treatment alone versus the other treatments; instead, we confirmed a significant increase in mortality related to its discontinuation. Treatment stop might be due to several reasons, such as uncertainties about possible adverse events, risk of interactions with other drugs, and possibly missing interactions between care managing physicians and referring hematologist. This is a potential bias in our analysis; however, a lot of effort was put into adjusting for all measured known confounders, which led us to perform a sensitivity analysis by PS matching which confirmed the previous findings.

The biological plausibility of this effect may be related to the devastating enhancement of inflammation ("cytokine storm") that follows the abrupt suspension of Ruxo, leading to the "ruxolitinib discontinuation syndrome", which in turn may lead to fatal clinical complications and multiorgan failure $[13,14]$. On the other hand, in observational studies, Ruxo itself has proven effective in improving survival in COVID-19 patients from the general population [15-17].

The main constraint of this study is the limited number of patients and, consequently, of events; this holds particularly true for the discontinued Ruxo group, which only consisted of 11 patients. However, we believe this work suggests that Ruxo treatment in MPN patients who are already assuming it at COVID-19 diagnosis should be continued, in agreement with the ASH recommendations (https://www.hema tology.org/covid-19/covid-19-and-myeloproliferative-

neoplasms). Of course, we acknowledge that this is a limitation that can hardly be avoided when dealing with both a rare condition, such as the MPN, and an exceptional situation of emergency, such as the COVID-19 crisis. Moreover, we stress that this study was originally designed to assess mortality and other outcomes in a vulnerable subpopulation of patients with COVID-19, and as such has not adequate power to test specific hypotheses about treatment effects. Yet, the effect of Ruxo discontinuation in our analysis was striking and robust to multiple confounders correction and sensitivity analysis.

This preliminary observation is, in our opinion, worthy of dissemination amongst patients and clinician communities, although it needs to be confirmed in a study with a longer follow-up and a sample size adequate to test the hypothesis. Efforts are currently undergoing to put in motion an observational study that meets these requirements.

Acknowledgements The study was supported by a research grant from the COVID " $3 \times 1$ project", BREMBO S.p.A., Bergamo, Italy.

Author contributions TB conceived and designed the study, supervised the analysis, and wrote the paper. $\mathrm{CH}$, AMV, AR, VDS, AAL revised the study and contributed to manuscript writing. AM directed the project. AC, AG, AF performed statistical analysis. AAL, AI, GR, EE, MMAC, MGK, JJK, FP, GB, VGG, MLF, MAF, CMM, ER, SO, PP, MBo, KSQC, MSS, GCT, MAS, FL, AP, BNE, AA, EMM, SK, MR, BC, JCHB, ELA, BXC, PG, MG, DC, RD, FC, BB, LB, NCG, $\mathrm{MBe}, \mathrm{SB}$ collected data. All authors revised and approved the final version of the manuscript.

\section{Compliance with ethical standards}

Conflict of interest The authors declare that they have no conflict of interest.

Publisher's note Springer Nature remains neutral with regard to jurisdictional claims in published maps and institutional affiliations. 


\section{References}

1. Richardson S, Hirsch JS, Narasimhan M, Crawford JM, McGinn T, Davidson KW, et al. Presenting characteristics, comorbidities, and outcomes among 5700 patients hospitalized with COVID-19 in the New York City Area. JAMA. 2020;323:2052-9.

2. Xu J, Wang L, Zhao L, Li F, Liu J, Zhang L, et al. Risk assessment of venous thromboembolism and bleeding in COVID-19 patients. Research Square 2020. On line publication. https://doi. org/10.21203/rs.3.rs-18340/v1.

3. Carsana L, Sonzogni A, Nasr A, Rossi RS, Pellegrinelli A, Zerbi $\mathrm{P}$, et al. Pulmonary post-mortem findings in a series of COVID-19 cases from northern Italy: a two-centre descriptive study. Lancet Infect Dis. 2020;20:1135-40.

4. Breccia M, Piciocchi A, De Stefano V, Finazzi G, Iurlo A, Fazi P, et al. COVID-19 in Philadelphia-negative myeloproliferative disorders: a GIMEMA survey. Leukemia. 2020;34:2813-4.

5. Palandri F, Piciocchi A, De Stefano V, Breccia M, Finazzi G, Iurlo A, et al. How the coronavirus pandemic has affected the clinical management of Philadelphia-negative chronic myeloproliferative neoplasms in Italy-a GIMEMA MPN WP survey. Leukemia. 2020;34:2805-8.

6. Barbui T, Tefferi A, Vannucchi AM, Passamonti F, Silver RT, Hoffman R, et al. Philadelphia chromosome-negative classical myeloproliferative neoplasms: revised management recommendations from European LeukemiaNet. Leukemia. 2018;32:1057-69.

7. Rosenbaum PR, Rubin DB. The central role of the propensity score in observational studies for causal effects. Biometrika. 1983;70:41-55

8. Vannucchi AM, Sordi B, Morettini A, Nozzoli C, Poggesi L, Pieralli F, et al. Compassionate use of JAK1/2 inhibitor ruxolitinib for severe COVID-19: a prospective observational study. Leukemia. 2020;19:1-13.
9. Pemmaraju N, Yu J, Parasuraman S, Paranagama D, Kish J, Visaria J, et al. Ruxolitinib (RUX) retreatment in patients (Pts) with myelofibrosis (MF): Real-world evidence on pt characteristics and outcomes. J Clin Onc. 2020;38:e19535.

10. Passamonti F, Cattaneo C, Arcaini L, Bruna R, Cavo M, Merli F, et al. Clinical characteristics and risk factors associated with COVID-19 severity in patients with haematological malignancies in Italy: a retrospective, multicentre, cohort study. Lancet Haematol. 2020;7:e737-45.

11. Onder G, Rezza G, Brusaferro S. Case-fatality rate and characteristics of patients dying in relation to COVID-19 in Italy. JAMA. 2020;323:1775-6.

12. Posso M, Comas M, Román M, Domingo L, Louro J, González C, et al. Comorbidities and mortality in patients with COVID-19 aged 60 years and older in a university hospital in Spain. Arch Bronconeumol. 2020;S0300-2896:30215-5.

13. Tefferi A, Pardanani A. Serious adverse events during ruxolitinib treatment discontinuation in patients with myelofibrosis. Mayo Clin Proc. 2011;86:1188-91.

14. Coltro G, Mannelli F, Guglielmelli P, Pacilli A, Bosi A, Vannucchi AM. A life-threatening ruxolitinib discontinuation syndrome. Am J Hematol. 2017;92:833-8.

15. La Rosée F, Bremer HC, Gehrke I, Kehr A, Hochhaus A, Birndt $\mathrm{S}$, et al. The Janus kinase $1 / 2$ inhibitor ruxolitinib in COVID-19 with severe systemic hyperinflammation. Leukemia. 2020;34:1805-15.

16. Heidel F, Hochhaus A. Holding CoVID in check through JAK? The MPN-approved compound ruxolitinib as a potential strategy to treat SARS-CoV-2 induced systemic hyperinflammation. Leukemia. 2020;34:1723-5.

17. Koschmieder S, Jost E, Cornelissen C, Müller T, Schulze-Hagen M, Bickenbach J, et al. Favorable COVID-19 course despite significant comorbidities in a ruxolitinib-treated patient with primary myelofibrosis. Eur J Haematol. 2020;105:655-8.

\section{Affiliations}

Tiziano Barbui $\mathbb{1}^{1} \cdot$ Alessandro Maria Vannucchi ${ }^{2} \cdot$ Alberto Alvarez-Larran $^{3}$ - Alessandra lurlo $\mathbb{B}^{4}$.

Arianna Masciulli ${ }^{1}$ - Alessandra Carobbio $\mathbb{1}^{1}$ - Arianna Ghirardi ${ }^{1}$ - Alberto Ferrari ${ }^{1}$ - Giuseppe Rossi ${ }^{5}$ Elena Elli ${ }^{6}$. Marcio Miguel Andrade-Campos ${ }^{7}$. Mercedes Gasior Kabat ${ }^{8}$ - Jean-Jaques Kiladjian ${ }^{9}$. Francesca Palandri ${ }^{10}{ }^{10}$ Giulia Benevolo $^{11}$ - Valentin Garcia-Gutierrez ${ }^{12}$ Maria Laura Fox ${ }^{13} \cdot$ Maria Angeles Foncillas $\mathbb{1}^{14}$. Carmen Montoya Morcillo ${ }^{15}$. Elisa Rumi ${ }^{16}{ }^{16}$ Santiago Osorio ${ }^{17}$. Petros Papadopoulos ${ }^{18}{ }^{18}$. Massimiliano Bonifacio $\mathbb{1}^{19}$ - Keina Susana Quiroz Cervantes ${ }^{20}$ - Miguel Sagues Serrano ${ }^{21}$ • Gonzalo Carreno-Tarragona $\mathbb{1}^{22} \cdot$ Marta Anna Sobas ${ }^{23} \cdot$ Francesca Lunghi $^{24} \cdot$ Andrea Patriarca $^{25}$. Begona Navas Elorza ${ }^{26}$. Anna Angona ${ }^{27}$. Elena Magro Mazo ${ }^{28}$. Steffen Koschmieder $\mathbb{1}^{29} \cdot$ Marco Ruggeri ${ }^{30}$. Beatriz Cuevas $^{31}$ - Juan Carlos Hernandez-Boluda $\mathbb{1}^{32}$ - Emma Lopez Abadia ${ }^{33}$ • Blanca Xicoy Cirici ${ }^{34}$. Paola Guglielmelli $\mathbb{D}^{2}$ - Marta Garrote ${ }^{3}$ - Daniele Cattaneo ${ }^{4} \cdot$ Rosa Daffini $^{5} \cdot$ Fabrizio Cavalca $^{6}$ - Beatriz Bellosillo ${ }^{7}$.

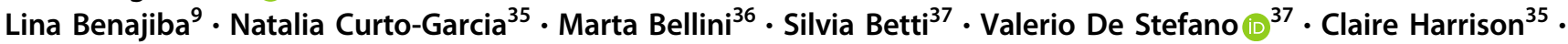
Alessandro Rambaldi $\mathbb{D}^{36,38}$

1 FROM Research Foundation, Papa Giovanni XXIII Hospital, Bergamo, Italy

2 Center Research and Innovation of Myeloproliferative Neoplasms (CRIMM), Department of Experimental and Clinical Medicine, Azienda Ospedaliera Universitaria Careggi, University of Florence, Florence, Italy

3 Hospital Clinic de Barcelona, Barcelona, Spain
4 Hematology Division, Foundation IRCCS Ca' Granda Ospedale Maggiore Policlinico, Milan, Italy

5 Spedali Civili, Brescia, Italy

6 Ospedale San Gerardo, ASST Monza, Monza, Italy

7 Hospital del Mar, Barcelona, Spain

8 Hospital Universitario la Paz, Madrid, Spain 
9 Hospital Saint-Louis, Paris, France

10 Azienda Ospedaliero-Universitaria di Bologna, Via Albertoni 15, Bologna, Italia

11 AOU Città della Salute e della Scienza di Torino, Torino, Italy

12 Hospital Ramon y Cajal, IRYCIS, Madrid, Spain

13 Department of Hematology, Vall d'Hebron Institute of Oncology (VHIO), Vall d'Hebron Hospital Universitari, Vall d'Hebron Barcelona Hospital Campus, C/ Natzaret, 115-117, 08035 Barcelona, Spain

14 Hospital Universitario Infanta Leonor, Madrid, Spain

15 Hospital General Universitario de Albacete, Albacete, Spain

16 Department of Molecular Medicine, University of Pavia, Pavia, Italy

17 Hospital Gregorio Maranon, Madrid, Spain

18 Hospital Clinico San Carlos, Madrid, Spain

19 Ospedale Policlinico “G.B. Rossi”, Borgo Roma, Verona, Italy

20 Hospital Universitario de Mostoles, Madrid, Spain

21 ICO L'Hospitalet-Hospital Moises Broggi, Sant Joan Despì, Barcelona, Spain

22 Hospital Universitario 12 de Octubre, Madrid, Spain
23 Department of Hematology, Blood Neoplasms and Bone Marrow Transplantation, Wroclaw Medical University, Wroclaw, Poland

24 IRCCS Ospedale San Raffaele, Milano, Italy

25 AOU Maggiore della Carità, Novara, Italy

26 Hospital Moncloa, Madrid, Spain

27 ICO Girona Hospital Josep Trueta, Girona, Spain

28 Hospital Universitario Principe de Asturias, Alcalà de Henares, Madrid, Spain

29 Department of Hematology, Oncology, Hemostaseology, and Stem Cell Transplantation, Faculty of Medicine, RWTH Aachen University, Aachen, Germany

30 Ospedale San Bortolo, Vicenza, Italy

31 Hospital Universitario de Burgos, Burgos, Spain

32 Hospital Clinico Universitario, INCLIVA, Valencia, Spain

33 Hospital General de Elche, Elche (Alicante), Spain

34 Hospital Germans Trias i Pujol, Badalona (Barcelona), Spain

35 Guy's and St. Thomas' NHS Foundation Trust, London, UK

36 ASST Papa Giovanni XXIII, Bergamo, Italy

37 Fondazione Policlinico “A. Gemelli” IRCCS, Rome, Italy

38 Università degli Studi di Milano, Milano, Italy 